\title{
SEPARATION THEOREMS AND THEIR RELATION TO RECENT DEVELOPMENTS IN ANALYSIS SITUS*
}

\author{
BY J. R. KLINE
}

1. Introduction. The study of those properties of point sets which are invariant under (1-1) continuous transformations form the subject matter of analysis situs. It is the purpose of the present paper to discuss one of the fundamental invariants of this field, that of a point set being separated by various of its subsets.

A set is said to be connected in the weak sense, if it is impossible to express it as the sum of two mutually separated sets, that is, two sets that are mutually exclusive and neither of which contains a limit point of the other one. A set $M$ is said to be connected in the strong sense if, for every two points $P$ and $Q$ of $M$, there is in $M$ a closed and connected subset containing both $P$ and $Q$. If to the set $K$, consisting of $y=\sin (1 / x),[0<x \leqq 1 / \pi]$, we add the origin, we obtain a set which is connected in the weak sense but not in the strong sense. A set $S$ is disconnected in the weak sense by one of its subsets $T$, in case $S-T$ is not connected in the strong sense; it is disconnected in the strong sense in case $S-T$ is not connected even in the weak sense. Thus every set $T$ which disconnects $S$ in the strong sense also disconnects it in the weak sense but the converse is not true. Thus the set $K^{\prime}$, composed of the above set $K$ together with the $y$-axis between $(0,+1)$ and $(0,-1)$ is disconnected in the weak sense by the removal of the origin but is not so disconnected in the strong sense. It is interesting to note that according to a theorem of R. L. Wilder, (1) if one limits himself to separating sets $T$ which are closed, then if $S$ is a continuous curve, $T$ disconnects $S$ in the weak sense only if it disconnects it in

* An address delivered at the meeting of this Society on May 7, 1927, in New York City, by invitation of the Program Committee. 
the strong sense; and (2) continuous curves are the only continua* for which the two types of separation by closed sets are equivalent. $\dagger$

The theorems of analysis situs are divided roughly into those that are concerned with continuity considerations and those of a combinatorial character. In the present paper we shall attempt to correlate the numerous important and rather widely scattered results that have been obtained in the field which deals with continuity considerations. The theory has been developed most extensively in two dimensions. However, while our material is arranged according as the separating sets and the sets separated are two-dimensional continua of various types, we shall always point out the analogous results in three or more dimensions and attempt to point out some of the difficulties in the cases where the extensions have not been made.

2. $S$ is a Euclidean Plane and $T$ is a Continuous Curve. $\$$ A notable step in the development of modern mathematics was made by Jordan when he expressed the necessity for a proof of the statement that every plane simple closed curve $\S$ divides its plane into an inside and an outside region

* A set which is both closed and connected will be called a continuum.

$\dagger$ See R. L. Wilder, Concerning continuous curves, Fundamenta Mathematicae, vol. 7 (1925), Theorem 8, p. 373. This paper will hereafter be referred to as W. F.

$\ddagger$ A continuous curve is the set of points whose coordinates can be represented as $x=f(t)$ and $y=g(t)$, where $f(t)$ and $g(t)$ are continuous, single valued functions of $t, 0 \leqq t \leqq 1$. A very useful point set characterization of a continuous curve was given by Hahn, when he proved that a necessary and sufficient condition that a closed and connected point set be a continuous curve is that it be connected im kleinen at all its points. A set of points is said to be connected im kleinen at a point $P$ if, whenever $C$ is a circle having $P$ as center, there exists a concentric circle $\bar{C}$ such that if $Q$ is a point of the set within $\bar{C}$ then $P$ and $Q$ lie together in some connected subset of the set which lies in $C$. See Hans Hahn, Mengentheoretische Characterisierung der stetige Kurve, Wiener Sitzungsberichte, vol. 123, Part IIa, pp. 24332489. For other characterizations of the continuous curve from a point set standpoint see R. L. Moore, A report on continuous curves from the viewpoint of analysis situs, this Bulletin, vol. 29 (1923), pp. 289-302.

$\S$ If $A$ and $B$ are distinct points, then a simple continuous arc from $A$ to $B$ is defined by Lennes as a closed connected set containing $A$ and $B$ but 
of which it is the common boundary. Jordan also supplied a proof of this theorem. However, his proof is unsatisfactory in many respects, particularly because he assumes the theorem for the case where the simple closed curve is a simple polygon.* The theorem was first proved completely by Veblen. $\dagger$ Numerous other proofs of the Jordan curve theorem have been given by other mathematicians, among whom we might mention Brouwer, Schoenflies, Alexander, de la Vallée Poussin, Bliss, Ames, Winternitz, and others. $\ddagger$ The simple closed curve, in addition to dividing its plane into two domains of which it is the common boundary, has the property that each point of the curve is approachable from both domains. $\$$ Schoenflies has proved the following theorem which is commonly known as the converse of the Jordan curve theorem:

Suppose $K$ is a closed bounded set of points lying in a euclidean plane $S$ and $S-K$ is the sum of two sets $K_{1}$ and $K_{2}$, which are mutually exclusive and are such that

(1) If $P$ and $Q$ are any two points of $K_{i}(i=1,2)$, then there exists an arc from $P$ to $Q$ entirely in $K_{i}$;

(2) An arc from a point of $K_{1}$ to a point of $K_{2}$ must contain at least one point of $K$;

containing no proper connected subset that contains both $A$ and $B$. A subset is a proper subset if its complement in the set is not vacuous. See $\mathrm{N}$. J. Lennes, Curves in non-metrical plane analysis situs with an application in the calculus of variations, American Journal, vol. 33 (1911), pp. 287-326. A simple closed curve is the sum of two arcs $A X B$ and $A Y B$ which have no points in common other than $A$ and $B$. A simple closed curve may also be defined as the set of all points which can be put into (1-1) continuous correspondence with the points of the circle.

* See C. Jordan, Cours d'Analyse, Paris, 2d edition, p. 92.

$\dagger$ See O. Veblen, Theory of plane curves in non-metrical analysis situs, Transactions of this Society, vol. 6 (1905), pp. 83-98.

$\ddagger$ For a complete list of proofs of this theorem see the article by Rosenthal in the Encyklopädie der Mathematischen Wissenschaften, $\mathrm{Bd} . \mathrm{II}^{3}$, Heft 7.

$\S$ A set of points $M$ is said to be approachable from a domain $D$, if whenever $P$ is a point of $M$ and $Q$ is a point of $D$, then there exists an arc $P Q$, which lies except for $P$ entirely in $D$. 
(3) If $P$ is a point of $K$ and $Q$ is a point not in $K$, then there exists an arc from $P$ to $Q$, lying except for $P$ entirely in $S-K .{ }^{*}$

R. L. Moore has proved that an open curve, that is, the set of points in (1-1) continuous correspondence with a straight line, divides its plane into two regions, similar to those made by the simple closed curve in the bounded case. $\dagger$ A proof of the converse theorem for the unbounded case has been given by the author of the present paper. $f$

Brouwer has proved the $n$-dimensional Jordan curve theorem, that is, that if $T$ is a set of points in (1-1) continuous correspondence with the set $x_{1}^{2}+x_{2}^{2}+x_{3}^{2}+\cdots+x_{n}^{2}=1$, lying in euclidean space of $n$ dimensions, then $T$ divides $S$ into two regions, of which it is the common boundary. $\S$ Veblen has proved that $n$-space is decomposed by a polyhedron. Veblen's proof does not use continuity considerations, in that he bases his proof on axioms I-VIII of his thesis, together with axioms which insure the existence of $n+1$ points not all lying in the same $(n-1)$-space and an $n$-dimensional closure axiom. No assumption of the archimedean axiom or the axiom of continuity is made.\| In this connection there is work of an extremely fundamental nature which has been done by J. W. Alexander. $T$

* See Schoenflies, Ueber einen grundlagenden Satz der Analysis Situs, Göttinger Nachrichten, 1902, p. 185.

† See R. L. Moore, On the foundations of plane analysis situs, Transactions of this Society, vol. 17 (1916), pp. 131-164. An open curve is defined, by Moore, from a point set standpoint, as a closed connected set of points $M$ such that if $P$ is any point of the set then $M-P$ is the sum of two mutually separated connected sets.

¥ See my paper, The converse of the theorem concerning the division of a plane by an open curve, Transactions of this Society, vol. 18, pp. 177-184. In a paper presented to the Society at the meeting in Chicago at Christmas 1925, P. M. Swingle has shown that in the presence of the other conditions, condition (1) may be omitted in both Schoenflies' theorem and mine. For an abstract of Swingle's paper see this Bulletin, vol. 32 (1926), p. 110.

$\S$ See L. E. J. Brouwer, Mathematische Annalen, vol. 71 (1912), p. 314.

$\|$ See O. Veblen, On the decomposition of $n$-space by a polyhedron, Transactions of this Society, vol. 13 (1912).

I See $A$ proof and extension of the Jordan-Brouwer separation theorem, Transactions of this Society, vol. 23 (1922), pp. 333-349. 
The converse of the Jordan curve theorem does not hold in three dimensions; this can very easily be seen if one considers the point set obtained from a sphere by deforming it continuously so that diametrically opposite points are brought together along a diameter. The set thus obtained will have the property of dividing three-space into just two domains of which it is the common boundary and such that each point is approachable from both domains; the set cannot be put into (1-1) continuous correspondence with a sphere. It seems probable that, in order to obtain a theorem analogous to the converse theorem of Schoenflies for two dimensions, one will be forced to use a stronger type of approachability. This appears likely because in the proof of Schoenflies' theorem very strong use is made of the fact that if $A$ and $B$ are points of the separating set and $A X B$ and $A Y B$ are arcs lying except for $A$ and $B$ entirely in the interior and exterior, respectively, then the sum of $A X B$ and $A Y B$ divides the space. This division does of course not hold in three dimensions and it appears probable that one will need an approachability, that will at least have an analogous separation property to that possessed by the arcs in the plane.

Schoenflies next set himself the problem: What will happen if one immerses a continuous curve in a euclidean space of two dimensions? Of course it is not necessary that the continuous curve shall divide the plane, for consider merely the case where we have a square plus its interior.* Schoenflies shows that if a continuous curve divides its plane then (1) there are in the complement of the continuous curve $M$ at

* That a continuous curve should fail to divide its plane, it is necessary and sufficient that every maximal cyclic curve of the set should be a simple closed curve plus its interior. A continuous curve $M$ is said to be a cyclic curve if every pair of points belonging to the curve lie on some simple closed curve which is a subset of $M$. A cyclic curve $K$ is said to be a maximal cyclic curve of the continuous curve $M$, if $K$ is not a proper subset of any other cyclic curve belonging to $M$. See G. T. Whyburn, this Bulletin, vol. 33, p. 520 . 
most a finite number of maximal connected subsets* of diameter greater than a preassigned $e \dagger,[e>0]$, and (2) every point on the boundary of a complementary domain is approachable from all sides from the domain. $\ddagger$ A condition that is equivalent to Schoenflies' condition (2) is furnished by assuming that the boundary of a complementary domain is regularly accessible, that is, if for every point $P$ on the boundary of a complementary domain $D$ and every preassigned $e[e>0]$, there exists a $\delta_{\epsilon}$ such that if $X$ is a point of $D$ at a distance from $P$ less than $\delta_{\epsilon}$, then $X$ and $P$ may be joined by an arc in $D$ except for $P$ and of diameter less than $e . \S$ These two properties tell us about points of the continuous curve which are on the boundary of a complementary domain. But it is evident that there are continuous curves in which there are points not on the boundary of any complementary domain. Let $[N]$ be the set of all points of the continuous curve $M$ not on the boundary of any complementary domain. Then (a) if $P$ is a point of $[N]$, Whyburn shows that for every $e>0$, there is in $M$ a simple closed curve of diameter less than $e$ which encloses $P \|$, and (b) $M-[N]$,

* A set $K$ is said to be a maximal connected subset of a set $M$ if $K$ is a connected subset of $M$ but is not a proper subset of any other connected set in $M$.

$\dagger$ It is interesting that this is still true if $S$, the euclidean plane of the hypothesis, is replaced by any plane continuous curve and $T$ is a subcontinuous curve of $S$. It is not true, however, if $S$ is a continuous curve in three dimensions. See W. L. Ayres, Concerning continuous curves and correspondences, Annals of Mathematics, vol. 28 (1927), pp. 396-399.

$\ddagger$ Suppose $P$ is a point on the boundary of a complementary domain $D$ of the continuous curve $M$, while $A$ and $B$ are points of this boundary which are distinct from $P$. Let $A X B$ be an arc lying except for its end points entirely within $D$ while $D_{1}$ is any one of the subdomains of $D$ made by $A X B$ which has $P$ on its boundary. Then, if for every point $Q$ of $D_{1}$, there is an arc from $P$ to $Q$ lying entirely except for $P$ and $Q$ in $D_{1}$, then $P$ is said to be approachable from all sides. See Schoenflies, Die Entwicklung der Lehre von Punktmannigfaltigkeiten, zweiter Teil, Leipzig, 1908, p. 237, for a proof that these two conditions form a necessary and sufficient set that a subcontinuum of a euclidean plane be a continuous curve.

$\S$ See G. T. Whyburn, Concerning continua in the plane, Transactions of this Society, vol. 29 (1927), p.370. This paper will hereafter be referred to as W. T.

\| See G. T. Whyburn, this Bulletin, vol. 33 (1927), p. 262. 
that is, the sum of the boundaries of the complementary domains of $M$, is strongly connected if and only if $[N]$ does not separate the plane even in the weak sense.* If we have only condition (1) of Schoenflies' theorem, then while result (b) is still true, the statement (a) must be modified because we can only say that for every $P$ of $[N]$ and every $d>0$, there exists a continuum (not necessarily a simple closed curve), of diameter less than $d$ separating $P$ from all the points of the plane at a distance from $P$ greater than $d$, and $M$ is connected $i m$ kleinen at $P$. Thus whenever condition (1) of Schoenflies' theorem holds and (2) fails, then all the points at which the continuum fails to be connected im kleinen, lie on the boundary of some complementary domain. The conditions of Schoenflies, while necessary and sufficient that a two-dimensional continuum be a continuous curve, are neither necessary nor sufficient for a continuum in euclidean space of three dimensions. This has been shown by R. L. Moore. $\dagger$

Let us now consider two points $A$ and $B$ ( $A$ and $B$ may or may not be points of the continuous curve $M$ ) which have the property that every arc from $A$ to $B$ contains a point of $M$, different from $A$ and $B$. Under these circumstances (a) there is a simple closed curve lying in $M$ which separates $A$ from $B \ddagger$ and (b) while every arc from $A$ to $B$ must contain at least one point of $M$, it is always possible to join $A$ and $B$ by an arc that does not separate $M . \S$ It follows from (a)

* See G. T. Whyburn, this Bulletin, vol. 33 (1927), p. 389.

† See R. L. Moore, Concerning the relation of a continuous curve to its complement in three dimensions, Proceedings of the National Academy, vol. 8 (1922), pp. 33-38.

$\ddagger$ This result was obtained for the case where both $A$ and $B$ belong to the complement of $M$, by R. L. Moore. See Concerning continuous curves in the plane, Mathematische Zeitschrift, vol. 15 (1922), p. 159. The case where $A$ and $B$ do not belong to the complement of $M$ was handled by C. M. Cleveland, Concerning points of a continuous curve that are not accessible from each other, Proceedings of the National Academy, vol. 13 (1927), pp. 275-276.

§ See R. L. Moore, Concerning paths that do not separate a continuous curve, Proceedings of the National Academy, vol. 12 (1926), p. 745. 
that no acyclic continuous curve* divides its plane. Wilder has shown that every subcontinuum of the boundary of a complementary domain of a continuous curve is itself a continuous curve. $\dagger$

The papers, which we have mentioned so far, have considered the continuum as given and have asked the question: What can be said of the complementary domains and their relation to the continuous curve and its subsets? Then there is also the inverse problem of having a domain given and asking under what conditions the boundary of a single domain shall be a continuous curve or some particular type of continuous curve. Carathéodory first obtained conditions under which the boundary of a single domain will be a simple closed curve. $\ddagger$ In his treatment, conditions are imposed both upon the boundary and on the relation of the domain to the boundary. In 1918, R. L. Moore found conditions on the domain, without any reference to the boundary, these conditions being necessary and sufficient that the boundary of the domain be a simple closed curve. The condition is that the domain be uniformly connected im kleinen, that is, for every $\epsilon>0$, there exists a $\delta$ such that if $X$ and $Y$ are two points of the domain at a distance from one another less than $\delta$, then they can be joined by a connected subset of the domain of diameter less than $\epsilon . \S \mathrm{He}$ afterwards proved that if one requires that the boundary of a simply connected domain be merely a continuous curve instead of specifying that it be the particular type of continuous curve known as a simple closed curve, then the condition of being uniformly connected im kleinen may be replaced by a weaker requirement, namely

* An acyclic continuous curve is a continuous curve that contains no simple closed curves. This term was first introduced by H. M. Gehman. Menger and a number of foreign writers call the same sets "Baumkurven."

$\dagger$ See W. F., loc. cit., Theorem 11, p. 361.

‡ See C. Carathéodory, Mathematische Annalen, vol. 73 (1912-1913), p. 366.

\$ See R. L. Moore, A characterization of Jordan regions by properties having no reference to their boundaries, Proceedings of the National Academy, vol. 4 (1918), pp. 364370. 
that of having the $S$-property, ${ }^{*}$ that is, that for every $e[e>0]$ the set may be expressed as the sum of a finite number of connected sets of diameter less than $e . \dagger$ Conditions equivalent to the assumption of the $S$-property for the domain have been given by Wilder and Whyburn but in both cases the conditions are of a different character from those of Moore in that they place restrictions on the boundary while Moore's are upon the domain alone. The condition of Wilder is that every connected subset of the boundary be connected in the strong sensef while Whyburn's is that every point of the boundary be accessible from all sides from $D$, the domain. $\$$ Whyburn also shows that a necessary and sufficient condition that a continuous curve $M$ be the bo undary of a connected domain is that, if $J$ denotes any simple closed curve in $M$, then (1) $J+I$ or $J+E$, where $I$ and $E$ denote respectively the interior and exterior of $J$, contain $M$ as a subset and (2) if $A$ and $B$ are any two points of $J$, then $M-(A+B)$ is not connected.\|

There is still a third type of problem that must be considered under the general heading of the separation of the plane by continuous curves. The papers which we have discussed have either (a) taken a given continuous curve and examined its complement or (b) considered a domain as given and hunted for conditions under which the boundary was a continuous curve. The problem in the second case is equivalent to that of being given a domain and asking under what conditions the maximal connected subsets of the complement are continuous curves. In both cases the separating set was either given or was found by taking certain limiting points of sets that were given. Let us now consider the problem where the sets to be separated are given and the

* See W. Sierpinski, Sur une condition pour qu'un continu soit une courbe jordanienne, Fundamenta Mathematicae, vol. 1 (1920), pp. 44-60.

$\dagger$ See R. L. Moore, Concerning connectedness im kleinen and a related property, Fundamenta Mathematicae, vol. 3 (1922), pp. 232-237.

$\ddagger$ See W. F., loc. cit., Theorem 19, p. 375 .

$\S$ See W. T., loc. cit., p. 370.

\| See W. T., loc. cit., Theorem 6, p. 380. 
dividing sets must be found and can in general not be found by merely taking limit points of sets that are already given.

In 1905 , Zoretti showed that if $K$ is a bounded maximal connected subset of a plane closed set $M$ and $\epsilon$ is any positive number, then there exists a simple closed curve $J$ enclosing $K$, passing through no point of $M$ and such that every point of $J$ is at a distance less than $\epsilon$ from some point of $K .^{*}$ It is evident that while every point of $J$ is at a distance less than $\epsilon$ from some point of $K$, this is not true of all points within $J$. For example $K$ might be a simple closed curve of diameter greater than $2 \epsilon$ which is in the set $M$. Moore shows that in the case where the set $K$ of our hypothesis does not separate its plane, then in addition to Zoretti's requirements, the simple closed curve $J$ may be so constructed as to have all points within it at a distance less than $\epsilon$ from some point of $K$. Thus with the use of these theorems, if $K$ and $H$ are mutually exclusive maximal connected subsets of the closed set $M$ and neither $K$ nor $H$ separates its plane, then we can get a simple closed curve containing one, excluding the other and passing through no point of $M$. If the continua $K$ and $H$ satisfy all the previous conditions except that instead of being mutually exclusive they may have a totally disconnected set $T$ in common, then we can construct a simple closed curve that encloses $K-T$ and has $H-T$ in its exterior whenever $K-T$ is connected. $\dagger$ Lubben has carried this work further and has obtained some very interesting results, one of the most important of which is the set of necessary and sufficient conditions for the separation of two

* See L. Zoretti, Sur les fonctions analytiques uniformes, Journal de Mathématiques, (6), vol. 1 (1905), pp. 9-11.

$\dagger$ For a proof of these theorems see R. L. Moore, Concerning the separation of point sets by curves, Proceedings of the National Academy, vol. 11 (1925), p. 469. The theorems mentioned have been for the bounded case. Moore also considers the case of being given a collection of unbounded continua and constructing open curves which shall separate sets of the collection. 
sets by a simple closed curve embodied in the following theorem:

If $K$ and $H$ are closed bounded point sets, whose common part $T$ is a totally disconnected set, while $K$ is a continuum, then a necessary and sufficient condition that there exist a simple closed curve containing a subset of $T$ but containing no point of $K+H-T$ and separating $K-T$ from $H-T$ is the following: $H-T$ (1) is a subset of a complementary domain of $K$ and (2) is not separated by $K$ near $T$.*

Practically nothing has been done with the corresponding problem in three dimensions. That the problem is an extremely complex one may be seen from an example in the work of Urysohn, in which he exhibits a closed totally disconnected set $T$ having the property that, while for every $\epsilon>0$, the set may be decomposed into a finite number of mutually exclusive parts each of diameter less than $\epsilon$ and each of these parts enclosed in a polyhedron not cutting any other polyhedron of the set, it is not always possible to choose these polyhedra so that each will be of genus zero. Indeed it may happen that the genus must become infinite as $\epsilon$ approaches zero. $\dagger$

3. $S$ is a Euclidean Plane and $T$ is a Continuum or Sum of Continua, not necessarily Continuous Curves. Here we are met with a situation that may be considerably more complicated. We might expect that a certain advantage would be gained by regarding the continuum as the sum of certain

* If $K, H$, and $T$ are point sets, then the statement that $H-K H$ is not separated by $K$ near $T$ means that if $P$ is a point and $C$ is a circle about $P$, then there exists a circle $C^{\prime}$ with the same center such that any pair of points $x$ and $y$ of $H-K H$ within $C^{\prime}$ can be joined by a connected point set which contains no points of $K$ and is entirely within $C$. See R. G. Lubben, The separation of plane point sets by curves, this Bulletin, vol. 32 (1926), p. 200. For abstracts of other results by Lubben on the same general problem see this Bulletin, vol. 32, pp. 13, 114, and 200.

$\dagger$ See P. Urysohn, Mémoire sur les multiplicités cantoriennes, Fundamenta Mathematicae, vol. 7 (1925), p. 121. Examples of a similar nature have been given by Antoine in his thesis, Sur l'homéomorphie de deux figures et de leurs voisinages. 
properly chosen subcontinua analogous to the case in the Jordan curve theorem, where a number of the proofs depend very strongly on the fact that the simple closed curve is the sum of two arcs which have no points other than their end points in common. However, this separation is not always possible, for we are forced to consider the case where our continuum may be indecomposable, that is, where it cannot be expressed as the sum of two distinct proper subcontinua. These continua were first introduced by Brouwer* and have been the subject of a great deal of work by Mazurkiewicz, Kuratowski, Knaster, Urysohn and others. With the aid of these continua examples have been given of continua which divide the plane into any finite number $n[n \geqq 3]$ or a countable infinity of domains, every point of the continuum being a boundary point of each of the domains. In this connection it is interesting to notice that a point set $K$ cannot be the common boundary of three mutually exclusive domains if there are in $K$ two points $A$ and $B$ each of which is accessible from all three of these domains. $\dagger$

Let us first consider the case where we shall regard our continuum as a single continuum and not as split up into various proper subsets. We will recall the result of $R$. L. Moore, who showed that whenever two points $A$ and $B$ are separated by the continuous curve $T$, then $T$ contains a simple closed curve which also separates $A$ from $B$. The simple closed curve has the property that, while it effects a separation of the plane between $A$ and $B$, no proper subset of the simple closed curve will separate the plane between $A$ and $B$. We shall say with Kuratowski that the point set $K$ is an irreducible separating set of the plane between $A$ and $B$,

* See L. E. J. Brouwer, The impossibility of a linear arrangement of the points of an irreducible continuum, Proceedings Academy, Amsterdam, vol. 14 (1911), p. 144. If $A$ and $B$ are distinct points, then an irreducible continuum from $A$ to $B$ is defined by Zoretti as a closed connected set of points containing $A$ and $B$ but containing no proper closed connected subset containing both $A$ and $B$. See Zoretti, Annales de l'École Normale, vol. 26 (1909).

† See G. T. Whyburn, this Bulletin, vol. 32 (1926), p. 200. 
whenever $K$ divides the plane into two or more mutually separated sets such that $A$ and $B$ belong to distinct sets but no proper subset of $K$ effects such a separation between $A$ and $B$. While $K$ may be an irreducible separating set of the plane between $A$ and $B$, there may be a different pair of points of the complement of $K$ which can be separated by a proper subset of $K$, as is illustrated by the following example:

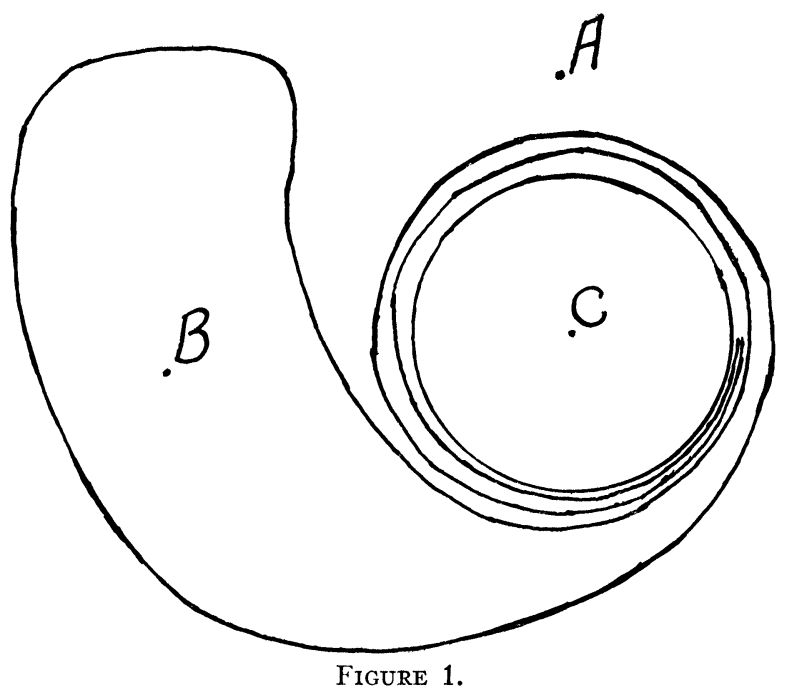

A set is said to be a completely irreducible separating set of the plane if the set $K$ divides the plane between some pair of points but no proper subset of $K$ divides the plane between any pair of points. Kuratowski has proved that:

(1) Every continuum separating the plane between $A$ and $B$ contains an irreducible separating set $K$ between $A$ and $B$ and this set $K$ is a continuum.

(2) Every continuum which divides its plane in to at most a finite number of complementary domains contains a completely irreducible separating set.*

* B. Knaster has given an example of a continuum which separates its plane into a countably infinite set of domains but contains no completely irreducible separating set. See Quelques coupures singulières du plan, Fundamenta Mathematicae, vol. 7 (1925), pp. 264-289. 
(3) Every completely irreducible separating set is either an indecomposable continuum or the sum of two continua each irreducible between the same pair of points. If the plane is divided into more than two domains by the irreducible separating set, then the set is either an indecomposable continuum or the sum of two such continua.*

Suppose we take a continuum, containing more than one point, which has the property that for every subset $K$ of $M$ that is connected, the set $M-K$ is connected in the weak sense. I have proved that every such continuum is a simple closed curve. $\dagger$ If instead of assuming that $K$ is any connected subset of $M$, we restrict ourselves to the case where $K$ is a closed connected subset of $M$ then

(1) I have shown that, if the plane continuum $M$ is not indecomposable and $M-K$ is connected in the weak sense, then $M$ must divide its plane and in case $M$ is a continuous curve, then $M$ must be a simple closed curve. $\ddagger$

(2) Kuratowski has shown that if in this case we assume that $M-K$ is connected in the strong sense, then $K$ must be a simple closed curve.§ Kuratowski's result is no longer true if we merely say that $M-K$ is connected in the weak sense. Consider the set composed of $y=\sin (1 / x)[0<x \leqq 1 / \pi]$, the $Y$-axis between $(0,+1)$ and $(0,-1)$ and an arc from $(0,-1)$ to $(1 / \pi)$ which has no point except its end points in common with the other sets. This set is connected in the weak sense after the removal of the set consisting of the portion of the

* For a proof of these results of Kuratowski, see Sur les coupures irréductibles du plan, Fundamenta Mathematicae, vol. 6 (1924), pp. 130-146. Results of a similar character were announced by G. A. Pfeiffer but never published. For an abstract of Pfeiffer's results, see this Bulletin, vol. 29 (1923), p. 151.

† See my paper Closed connected sets which remain connected upon the removal of certain connected subsets, Fundamenta Mathematicae, vol. 5 (1924), pp. 3-11.

¥ See my paper, Concerning the division of the plane by continua, Proceedings of the National Academy, vol. 10 (1924), pp. 176-177.

$\S$ C. Kuratowski, Contribution à l'étude de continus de Jordan, Funda menta Mathematicae, vol. 5 (1924), pp. 119-122. 
$Y$-axis between the origin and $(0,-1)$ but not connected in the strong sense.

R. L. Wilder has investigated the properties of sets $M$ which are connected and remain connected upon the removal of any connected subset, when one omits the condition that the set is closed. Such sets he calls quasi-closed curves. It is found that (1) if $A$ and $B$ are any two distinct points of $M$, then $M$ is the sum of two sets $M_{1}$ and $M_{2}$ each of which contains $A$ and $B$, is irreducibly connected from $A$ to $B$ and such that $M_{1}-(A-B)$ and $M_{2}-(A-B)$ are mutually separated sets, (2) $M$ separates the plane, and (3) if $M$ is connected im kleinen at all its points, then $M$ is a simple closed curve. The last result is particularly interesting as it gives a characterization of a simple closed curve in which the property of being closed is not assumed.*

Using the notion of prime parts of a continuumt, introduced by Hahn, R. L. Moore has further shown that if the plane continuum $M$ has more than one prime part and no one of its prime parts separates the plane, then a necessary and sufficient condition that $S-M$ should be the sum of just two mutually exclusive domains of which $M$ is the common boundary, is that for every subcontinuum $K$ of $M, M-K$ is connected. Under the same hypothesis, in order that $S-M$ shall be the sum of two mutually exclusive domains such that each prime part of $M$ will contain at least one limit point of each domain, it is necessary and sufficient that $M$ be a simple closed curve of prime parts in the sense that it is disconnected by the omission of any two of them which are not

* The results mentioned in (1) and (2) were presented at the International Mathematical Congress at Toronto and will appear in the Proceedings of that Congress. The one marked (3) is found in abstract in this Bulletin, vol. 32 (1926).

$\dagger$ If $P$ is a point of a continuum $K$, then the prime part of $K$, which contains $P$ is defined by Hahn as the set of all points $[X]$ such that for every positive number $e[e>0]$, there exists a finite number of irregular points of $K, X_{1}, X_{2}, X_{3}, \cdots, X_{n}$, such that $X_{1}$ is $P, X_{n}$ is $X$ and for every $i$ the distance $X_{i}$ to $X_{i+1}$ is less than $e$. A point is said to be an irregular point of $K$, if $K$ is not connected im kleinen at the point. See Hans Hahn, Ueber die irreducible Continua, Wiener Berichte, vol. 130 (1921). 
identical.* Whyburn has also proved that if a bounded closed set separates the plane in the weak sense but no proper subset does so, then the set must be a simple closed curve.

In this connection I wish also to call attention to an extremely interesting result of Urysohn, who has proved that if $K$ is the common boundary of two connected domains in space of three dimensions and $T$ is a totally disconnected subset of $K$ (that is, a set which contains no connected subsets other than single points) which is closed, then $K-T$ must be connected. $\dagger$

Let us now consider the case where $T$ is the sum of two or more continua. The first result in this direction is the famous Brouwer-Phragmen theorem, which states that if $A$ and $B$ are any two closed bounded sets, which have no points in common and neither of which disconnects the plane, then their sum cannot separate the plane. Mazurkiewicz in volume 3 of the Fundamenta has proved the unbounded case of the Brouwer-Phragmen theorem.

This fundamental result has been extended as follows.

(a) By a young Polish mathematician, Strasziewicz, who shows that the theorem also holds if we allow the common part to be connected. $\$$ This result is also contained implicitly in the work of Miss Mullikin.§

(b) By Miss Mullikin, who shows that if $A_{1}, A_{2}, A_{3}, \cdots$ is a countable set of closed sets, no one of which separates the plane, then their sum cannot do so.\|

* For the first of these results see Concerning the common boundary of two domains, Fundamenta Mathematicae, vol. 6 (1925), p. 203.

† See P. Urysohn, Mémoire sur les multiplicités cantoriennes, Fundamenta Mathematicae, vol. 7 (1925), pp. 103-119.

‡ See S. Strasziewicz, Ueber eine Verallgemeinerung des Jordan'schen Kurvensatz, Fundamenta Mathematicae, vol. 4 (1923), pp. 129-135.

$\S$ See Transactions of this Society, vol. 23 (1922).

|| Loc. cit., Theorem 3, pp. 148-155. A new proof of this interesting theorem has been given by Mazurkiewicz in Fundamenta Mathematicae, vol. 6, pp. 37-38. This theorem is closely connected with a theorem of mine Concerning the complement of a countable infinity of point sets of a certain type, this Bulletin, vol. 23, pp. 290-292. For extensions of my theorem see the abstract of a paper by R. L. Wilder, this Bulletin, vol. 33 (1927), p. 388. 
For the extension of the Brouwer-Phragmen result to $n$ dimensions there is an extremely interesting result by Alexandroff which may be stated as follows.

Suppose that in euclidean space of $n$ dimensions, we have two closed sets $F_{1}$ and $F_{2}$, neither of which decomposes the space. Then if the common part of these sets is of dimension* not greater than $n-3$ the sum $F_{1}+F_{2}$ cannot decompose the space $E_{n}$. The common frontier of two connected domains of $E_{n}$ is a cantorian multiplicity of dimension $n-1 . \dagger$

There is also a theorem closely related to the preceding extensions of the Brouwer-Phragmen theorem, in which we are given some further information about points in the sum. The theorem is one of Gehman's and is as follows. If $N$ is a closed bounded set consisting of a collection of connected sets $(E)$, each of which is a maximal connected subset of $N$, no one of which separates the plane $S$, and no more than a finite number are of diameter greater than any preassigned positive number, then $N$ cannot separate the plane and moreover if a point $P$ of a maximal connected subset $e$ of $E$ is accessible from $S-e$, then $P$ is accessible from $S-N$.

The theorems of the present section have been negative theorems in the sense that they have told us that when the individual sets have certain properties, then the sum cannot decompose the plane. When we look for theorems of a positive type, we are first met by the results of Janiszewski and Miss Mullikin, who working independently obtained the following important results.

(a) If $A$ and $B$ are closed and connected bounded sets, neither of which disconnects the plane, and have a common part which is not connected, then their sum must disconnect the plane.

* This concept will be defined in the next section of the present paper.

$\dagger$ See P. Alexandroff, Comptes Rendus, vol. 183, p. 722. A cantorian multiplicity is defined by Urysohn as a continuum which remains connected upon the removal of any closed set of dimension not greater than $n-2$.

¥ See H.M.Gehman, Concerning acyclic continuous curves, Transactions of this Society, vol. 29 (1927), pp. 558-560. 
(b) If the common part of $A$ and $B$ consists of exactly two maximal connected sets, then the plane is divided into exactly two parts by their sum.*

Knaster and Kuratowski have proved that these results remain true, if one only of the continua of the hypothesis is assumed bounded. When both are unbounded, then a necessary and sufficient condition that the sum should cut the plane when neither of the continua alone cut it, is that their common part should contain a maximal connected set which is bounded. $\dagger$ Strasziewicz $\ddagger$ has also proved that if the common part reduces to exactly $n$ maximal connected sets which are maximal, then there are exactly $n$ complementary domains. Still other results in this connection have been given by Mrs. Nikodym. $\S$

Rosenthal has considered a closely related question. He replaces the assumption that neither $A$ nor $B$ divides the plane by the following. Both $A$ and $B$ are continua which are irreducible between the same pair of points $a$ and $b$ and which have no other points in common. His principal result is that the complement of the sum with respect to its plane consists of two principal domains (Hauptgebiete), each of which has the entire sum as its boundary, and possibly of a number of secondary domains (Nebengebiete), each of which has its frontier wholly in either $A$ or $B . \|$ W. A. Wilson has generalized these results by showing that this theorem of Rosenthal remains true if the two continua $A$ and $B$ have in common two point sets $\alpha$ and $\beta$, where $\alpha \cdot \beta=0$, if

* See S. Janiszewski, Sur les coupures du plan faites par continus, Prace Matematyczne-Fisyczne, vol. 36 (1913). This paper is in Polish and the results contained therein were unknown to Miss Mullikin at the time of her work, which was published in the previously quoted paper, pp. 154-162.

$\dagger$ See Sur les continus non-bornés, Fundamenta Mathematicae, vol. 5 (1924), p. 36.

‡ See Strasziewicz, Ueber die Zerschneidung der Ebene, Fundamenta Mathematicae, vol. 7 (1925), pp. 159-188.

$\S$ See S. Nikodym, Sur les coupures du plan, Fundamenta Mathematicae, vol. 7 (1925), pp. 14-23.

|| See A. Rosenthal, Teilung der Ebene durch irreducible Kontinua, Sitzungsberichte der Münchener Akademie, 1919, pp. 91-109. 
each of the continua $A$ and $B$ is irreducible between any point $a$ of $\alpha$ and any point $b$ of $\beta$, and both $A$ and $B$ are decomposable or one of them is indecomposable and the other is not the union of two indecomposable continua. He also shows that the frontier of any secondary domain is part of either a continuum of condensation, an indecomposable continuum, a pair of indecomposable continua, or the union of an indecomposable continuum and a continuum of condensation.*

When we are working in three dimensions, the conditions of Janiszewski and Miss Mullikin are neither necessary nor sufficient. That the condition is not necessary may be seen if we consider the sets $A$ and $B$, where $A$ is the set composed of the great circle $g$ of the sphere $K$ plus one of the hemispheres cut off by $g$, while $B$ is the other hemisphere plus $g$. That the conditions are not sufficient may be seen by considering the set composed of two arcs $A X B$ and $A Y B$, which have no point in common other than $A$ and $B$. In the ninth volume of the Fundamenta Mathematicae, Mazurkiewicz and Strasziewicz have obtained conditions, which are sufficient but not necessary that the sum of two sets, neither of which separates three-space, should separate the same. Let us first introduce several definitions.

To every point $t$ of the interval $t_{1} \leqq t \leqq t_{2}$, we make correspond a point $X(t)$ and suppose that the function $X(t)$ is continuous for the interval under consideration. This defines a continuous curve, which we shall call closed if it is true that $X\left(t_{1}\right)$ and $X\left(t_{2}\right)$ are the same. Let $B$ be a closed point set having no points in common with the closed continuous curve $M$. Then we shall say that $M$ is free with respect to $B$, if there exists a function of two variables $Y(t, \lambda)$ defined and continuous for $t_{1} \leqq t \leqq t_{2}$ and $0 \leqq \lambda \leqq 1$ where

(a) $Y(t, \lambda)$ has nothing in common with $B$ for all values of the variables,

(b) $Y\left(t_{1}, \lambda\right)=Y\left(t_{2}, \lambda\right)$,

(c) $Y(t, 1)=X(t)$, for all values of $t$ and $Y(t, 0)=x_{0}$, a

* See W. A. Wilson, this Bulletin, vol. 33 (1927), pp. 733-744. 
constant. In case this function does not exist, then the curve is said to be interlaced with $B$. A closed set in three dimensions is said to be interlaceable if there exists a closed curve that is interlaced with $B$.

On the basis of these definitions, the authors prove the following theorems.

Theorem I. If $A$ and $B$ are closed sets neither of which divides three-space between the points $P$ and $Q$ and if the common part $A B$ is not interlaceable then their sum does not divide threespace between $P$ and $Q . *$

THEOREM II. If $A$ and $B$ are closed sets neither of which cuts three-space, then their sum cuts three-space if their common part is interlaceable while neither of the sets is interlaceable. $\dagger$

4. General Dimension Theory. Up to the present we have considered our containing set to be a euclidean space of $n$ dimensions, that is, a space satisfying, let us say, Veblen's axioms for geometry with sufficient additional axioms to allow for the existence of $n+1$ points not all in the same $(n-1)$ space, together with an $n$-dimensional closure axiom. We shall, of course, wish to speak of various sets in this $n$-dimensional space as having a dimension $k$, where $k$ may assume all values from zero to $n$. Various mathematicians have considered this problem of assigning a dimension to subsets of euclidean space of $n$ dimensions. Of course, a theory based on a set of axioms for euclidean geometry is bound to have a special character due to the interplay of the special properties of ordinary space; and it is for this reason that the

* Alexandroff has recently pointed out that Theorem I is a special case of a theorem due to Alexander. See J. W. Alexander, loc. cit., p. 342. Using Alexander's methods and the notion of the Betti Numbers for a general closed set introduced by himself, Alexandroff has obtained interesting extensions of the Brouwer-Phragmen theorem and of the Janiszewski-Mullikin theorem. See P. Alexandroff, Zum verallgemeinerten Phragmen-Brouwer'schen Satz, Fundamenta Mathematicae, vol. 11 (1928), pp. 222-227.

$\dagger$ See S. Mazurkiewicz and S. Strasziewicz, Sur les coupures de l'espace, Fundamenta Mathematicae, vol. 9 (1927), pp. 205-211. 
founders of the recent developments in dimension theory, Menger and Urysohn, considered themselves immersed in a more general space and considered the problem of assigning a dimension to sets in general metric space.

Let us consider a set of elements, $E$, about which nothing is assumed as to their nature, and a non-negative function $r(x, y)$ satisfying the following conditions:

(1) $r(x, y)=r(y, x)$;

(2) $r(x, y)=0$, if and only if $x$ and $y$ are identical;

(3) $r(x, y) \leqq r(y, z)+r(z, x)$.

We shall assume that our space in addition to having the above distance function, which makes it a metric space, has the property of being compact, that is, every infinite set of distinct points has a limit point.

A rather complete survey of the early attempts to define dimension has been given by Menger,* in which he also shows how each of these attempts is unsatisfactory because it assigns a dimension to certain sets which is different from the dimension we would intuitively assign to it; for example the definition of Zoretti of a one-dimensional continuum as an irreducible continuum would assign the dimension one to the surface of a cube which can be a subset of an irreducible continuum in three dimensions. It is rather interesting to note that this problem received a satisfactory solution almost simultaneously at the hands of two young mathematicians, one a Russian, Urysohn, and the other an Austrian, Menger. The theories of these two men are equivalent for compact metric spaces. $\dagger$ The theory of Urysohn might be characterized as an im kleinen while that of Menger is an im grossen theory. We shall first take up the Urysohn theory. $\$$

* See K. Menger, Bericht über die Dimensionstheorie, Jahresbericht der Vereinigung, vol. 35 (1926), pp. 113-150.

$\dagger$ For an example of a space satisfying Hausdorff's four axioms for topological space and which contains a set that is two-dimensional according to the definition of Menger, but zero-dimensional by Urysohn's definition, see an article by L. W. Cohen, Comptes Rendus, 1927.

$\ddagger$ See P. Urysohn, loc. cit.; and also Fundamenta Mathematicae, vol.8 (1926). 
Let $P$ be a point of the set $C$ which lies in a compact metric space and $\epsilon$ any positive number. Then we shall say that we have an $e$-separation of $C$ at point $P$ if we have the following conditions satisfied:

(1) $C=A+B+D$, where $A, B$, and $D$ are mutually exclusive sets;

(2) $A$ contains $P$;

(3) $A+B$ is of diameter less than $\epsilon$;

(4) $A$ and $D$ are mutually separated sets.

We shall now define the dimension of a point set as follows:

(1) The dimension of the vacuous set shall be -1 ;

(2) A set shall be zero-dimensional at a point $P$ if $C$ can be separated at $P$ by a vacuous set, that is, by a set of dimension -1 ;

(3) The dimension of a point set shall be the greatest positive integer that occurs amongst the dimensions of its various points, (thus we may have sets whose dimension is not finite);

(4) Suppose all the dimensions up to and including the $(n-1)$ st have been defined; then we shall say that a set is of dimension $n$ at $P$ if

(a) The set may be $e$-separated at $P$ by a set of dimension less than $n$,

(b) The dimension of the set at $P$ is not less than $n$.

The definition of Menger is as follows: The space is at most $n$-dimensional if every two closed mutually exclusive sets of the set can be separated by a set which is at most $(n-1)$-dimensional. The definition given by Menger is practically the same as that which Brouwer gave in 1913, but which he carried no farther than showing that every subset of $R_{n}$ (that is, euclidean space of $n$ dimensions) which contains an open subset is $n$-dimensional, where an open subset is one whose complement is closed. The above definitions were discovered independently and without any knowledge of the definition of Brouwer. In both systems there are the necessary theorems to show that the dimension as defined leaves sets, to which our intuition would tell us to assign the dimen- 
sion $n$, with this dimension. As Menger points out, this definition is already foreshadowed by Euclid when he calls the line that whose boundary is points, the surface that whose boundary is lines, and the solid that whose boundary is surfaces.

To show that the dimension assigned to a point set is in accordance with our intuition and that no contradictions arise in applying it, a long series of theorems must be proved and in a number of cases the argument is very long and involved. The principal theorems follow:

(1) The dimension of a point set remains invariant under (1-1) continuous transformations.

(2) If $F_{1}, F_{2}, F_{3}, \cdots$ is a countable infinity of closed sets each of dimension $n$, then the sum is of dimension $n$.

(3) Every totally disconnected set which is closed is zerodimensional.

(4) The sum of a set of $n$ dimensions and one of $m$ dimensions is a set of dimension not more than $m+n+1$. With the use of this theorem it is proved that every $n$-dimensional set of a separable space (that is, a space where there is an everywhere dense countable set) is the sum of $n+1$ but not fewer zero-dimensional sets.

(5) Every closed domain of euclidean space of $n$ dimensions is n-dimensional.

This last theorem is proved on the basis of a remarkable theorem due to Lebesgue, which is as follows.

If each point of a domain of $n$ dimensions belongs to at least one of the closed sets $F_{1}, F_{2}, F_{3}, \cdots, F_{k}$, finite in number, and if the sets are sufficiently small, then there is a point common to at least $n+1$ of the sets; on the other hand whatever the domain $G$, and whatever be the diameter of the sets $F_{i}$, then it is always possible to decompose $G$ so that each point is not common to more than $n+1$ of the sets.*

* This theorem was first announced by Lebesgue in 1911 (Mathematische Annalen, vol. 70, pp. 166-168). The complete demonstration of the theorem was found for the first time in the work of Brouwer in Crelle's Journal, vol. 142 (1913). See also Lebesgue, Sur les correspondences entre les points de deux espaces, Fundamenta Mathematicae, vol. 2 (1921), p. 257. 
Still more remarkable is the result announced by Menger to the effect that every set of $k$ dimensions which is closed is a subset of some continuous curve lying in euclidean space of $2 k+1$ dimensions.*

5. Sis a Proper Subcontinuum of the Euclidean Plane and T is a Subset of $S$. For the sake of convenience we shall divide the material of this section as follows.

(a) Cut Points of Continua, that is, points of a continuum which separate it.

(i) Conditions for their Existence.

(ii) Properties of the Set $[C]$ of all Cut Points.

(iii) Characterization of Various Types of Continuous Curves by Properties of their Cut Points.

(b) Disconnection by Pairs of Points.

(c) The Menger Curve Theory.

(d) The Set $T$ is a Finite Number (or Countable Infinity) of Continua.

5a. Cut Points of Continua. (i) Conditions for their Existence. Let us consider three very simple continuous curves, the open curve, that is, the set of points in (1-1) continuous correspondence with the set of points on a straight line, the simple continuous arc from $A$ to $B$, and the set composed of a square plus its interior. In the first every point is a cut point, in the second every point other than $A$ and $B$ is a cut point, while in the third there is no cut point. When one is examining a continuum as to the existence or non-existence of cut points, it is necessary to examine only the boundaries of the complementary domains of the continuum, for according to a theorem due to G. T. Whyburn, a necessary and sufficient condition that a point $P$ of a continuum $M$ be a cut point of $M$ is that it be a cut point of the boundary of some complementary domain of $M . \dagger$ It one takes two particular

* See K. Menger, Allgemeine Räume und Cartesische Räume, Königliche Akademij, Amsterdam, vol. 29 (1927), Nos. 3 and 8.

$\dagger$ See W. T., loc. cit., p. 389. This theorem is an extension of a theorem published in 1925 by R. L. Moore, who showed that in order that a bounded continuous curve $K$ should have no cut point, it is necessary and sufficient 
points $A$ and $B$ of the continuous curve $M$, then that there be a point in $M$ which separates $A$ from $B$ in $M$, it is necessary and sufficient that every two simple continuous arcs in $M$ from $A$ to $B$ shall have a point in common different from $A$ and $B .^{*}$

In 1918 Mazurkiewicz showed that (1) every continuous curve $M$ which is bounded has at least two points which do not disconnect $M$ in the weak sense and (2) every continuous curve $M$ which contains a simple closed curve $J$ has the property that $J$ contains at most a countable set of points which disconnect $M$ in the weak sense. It will be noticed that the points of $J$ which disconnect $M$ do not disconnect $J . \dagger$ In these theorems the condition that $M$ be a continuous curve may be replaced by the weaker condition that $M$ be a continuum. This follows from two theorems of R. L. Moore to the effect that (1) every continuum whatsoever contains at least two points neither of which disconnect it in the strong sense and (2) no continuum $M$ (whether it be a continuous curve or not) contains a closed and connected set $K$ (whether $K$ is a simple closed curve or not) which contains an uncountable set of points each of which disconnects $M$ but not $K$. It is interesting to note that Zarankiewicz has pointed out that the demonstration of Moore's second theorem can be so modified as to apply to the case where $M$ is merely assumed to be connected (bounded or unbounded). The statement of Zarankiewicz's theorem is as follows. If $N$ is any connected

that the boundary of every complementary domain be a simple closed curve. See Moore, Concerning the common boundary of two domains, Fundamenta Mathematicae, vol. 6 (1925), p. 211.

* See W. L. Ayres, On the separation of points of a continuous curve by arcs and simple closed curves, this Bulletin, vol. 33 (1927), p. 266.

$\dagger$ See S. Mazurkiewicz, Un théorème sur les lignes de Jordan, Fundamenta Mathematicae, vol. 2 (1921), pp. 119-130.

$\ddagger$ See R. L. Moore, Concerning the cut points of continuous curves and other closed and connected sets, Proceedings of the National Academy, vol. 9 (1923), pp. 101-102. This paper will hereafter be referred to as M.C.P. 
subset of a connected set $C$, then the set of points of $N$ which cut $N$ without cutting $C$ is at most countable.*

(ii) Properties of the Set [C] of all Cut Points. A great deal of interesting work has been done on the set $[C]$ composed of all cut points of a given continuous curve, particularly as to the number of such cut points and various properties possessed by their subsets.

Zarankiewicz has shown that the set $[C]$ is a set $F_{\sigma}$, that is, the sum of a countable infinity of closed sets. $\dagger$ If we consider the subset $[B]$ of all points of $[C]$ such that for every point $b$ of $[B], M-b$ is neither connected or the sum of two connected sets, then $[B]$ is finite or countably infinite. $\ddagger$ This theorem is proved merely on the assumption that $M$ is connected. In case that $M$ is an acyclic continuous curve, the set $[B]$ becomes the set of branch points of $M$, and hence the theorem contains the result of Menger that the branch points of an acyclic continuous curve are at most countable. $\S$

G. T. Whyburn has proved in his thesis a number of interesting theorems concerning the properties of the subcontinua of $[C]$, the most striking of which are the following.

(1) If $[H]$ denotes the set of end points $\|$ of the continuum $M$ while $[T]$ is any countable subset of $M$, then every bounded

* See C. Zarankiewicz, Sur les points de division dans les ensembles connexes, Fundamenta Mathematicae, vol. 9 (1927), Theorem 9, p. 140. This paper will hereafter be referred to as Z.F.M.

† See Z.F.M., loc. cit., Theorem 17, p. 163.

$\ddagger$ See C. Kuratowski and C. Zarankiewicz, A theorem on connected sets, this Bulletin, vol. 33 (1927), pp. 571-575.

$\S$ See K. Menger, Ueber reguläre Baumkurven, Mathematische Annalen, vol. 96 (1926), p. 574. A point $p$ is said to be a branch point of an acyclic continuous curve if there exist in the curve three arcs $L_{1}, L_{2}$, and $L_{3}$ such that $p=L_{1} \cdot L_{2}=L_{2} \cdot L_{3}=L_{3} \cdot L_{1}$.

$\|$ If $M$ is a continuous curve, then an end point of $M$ is defined by Wilder as a point $P$ such that, whenever $a$ is an arc from $P$ to any point $P^{\prime}$ of $M$, then the set $M-(a-P)$ contains no connected subset which contains more than one point and contains $P$. See W.F., loc. cit., p. 358. As applied to continua in general, Whyburn defines the term end point as follows. The point $P$ is said to be an end point of the continuum $M$ provided it is true that if $N$ is any subcontinuum of $M$ which contains $P$, then $P$ is not a limit point of any connected subset of $M-N$. 
continuum which is a subset of $[C]+[H]+[T]$ is an acyclic continuous curve.*

A closely related result is that due to Zarankiewicz in which he shows that in a continuous curve $M$, if $K$ is any constituent of $\dagger[C]$, the set of cut points of $M$, then $\bar{K}$, that is, the set $K$ plus its limit points, is an acyclic continuous curve. $\neq$

(2) For every positive number e, $[C]$ does not contain more than a finite number of continua of diameter greater than $e$.

Zarankiewicz shows a slightly more general result in that he proves that you may replace "continua" by constituents and further shows that the constituents of diameter greater than $e$ lie on an acyclic continuous curve which is a subset of $M . \S$

(3) If $[C],[H]$ and $[N]$ denote, respectively, the cut points, end points and simple closed curves in $M$, then $M$ is the sum of these three sets.

(4) If $K$ is any connected subset of the set [C], then in order that $\bar{K}$ should be an acyclic continuous curve, it is necessary and sufficient that every point of $\bar{K}$ be either an end point or a cut point of $M$.

The result of Zarankiewicz mentioned under (1) above shows that for the case where $K$ is a maximal connected subset of $[C]$ which is connected in the strong sense, $\bar{K}$ is always an acyclic continuous curve.

* This result is Theorem 9 of W.T., loc. cit., p. 383. Whyburn's result is not only for the case where $M$ is a continuous curve but for the more general case where $M$ is any plane continuum. C.M. Cleveland announced the special case of Whyburn's result where the subset considered is a maximal connected subset of the cut points $[C]$ of a continuous curve. See this Bulletin, vol. 32 (1926), p. 420.

$\dagger$ According to Kuratowski, the constituent of a point $p$ in a point set $P$, is the set of all points of $P$ which lie together with $p$ in a closed connected subset of $P$.

$\ddagger$ See C. Zarankiewicz, Sur la structure d'un ensemble de points de division dans les continus de Jordan, Bulletin de l'Académie Polonaise des Sciences et des Lettres, 1926, p.362. This paper will be referred to as Z.P.B.

$\S$ See Z.P.B., loc. cit., Theorems 2 and 3. 
(5) If $K$ denotes the set of all cut points of the boundary of a complementary domain $D$ of a continuous curve $M$, then the sum of $D$ and $K$ is uniformly connected im kleinen.*

(6) Suppose $A$ and $B$ are two points of a continuous curve $M$ and $[K]$ denotes the set of all points which separate $A$ from $B$ in $M$. Under these conditions,

(i) $A+[K]+B$ is a closed set;

(ii) If $t$ is any arc of $M$ from $A$ to $B$ and $S$ is any maximal segment of $t-(A[K] B)$, then $M$ contains a maximal cyclic curve which contains $S$.

(7) If any cut point of $M$ is a point at which some continuum. of $M$ is not connected im kleinen, then $P$ is on some simple closed curve in $M$ and the set of all such points is countable. $\dagger$

(iii) Characterization of Various Types of Continuous Curves by Properties of their Cut Points. The first sets to be characterized by their cut points were simple continuous arcs. In 1916, Sierpinski showed that an arc is a bounded set, containing more than two points such that for every point of the set except two, the set is the sum of two closed sets which have only the point $P$ in common. $f$ In the Sierpinski characterization the boundedness assumption is essential. Moore has given another set of conditions in which the boundedness is not assumed but more information is required about the connectedness than was assumed by Sierpinski. Moore's theorem is to the effect that if the continuum $M$ contains two points $A$ and $B$ such that (1) $M-A$ and $M-B$ are connected, (2) $M-P$ is not connected if $A \neq P \neq B$, then $M$ is a simple continuous arc from $A$ to $B$.

\footnotetext{
* The results listed above are Theorems $9,14,22,13$, and 4 , respectively, of W.T.

$\dagger$ For results (6) and (7), see this Bulletin, vol. 33 (1927), p. 520.

¥ See S. Sierpinski, L'arc simple comme un ensemble de points dans l'espace à m dimensions, Annali di Matematica, (3), vol. 26, pp. 131-150. As R. L. Moore pointed out, it is implicitly assumed that the two exceptional points do not cut.
} 
If the boundedness is assumed then condition (1) may be omitted.*

An open curve, that is, the set of all points in (1-1) continuous correspondence with the straight line, is characterized as a continuum $M$ which, whenever $P$ is a point of the set, then $M-P$ is the sum of two mutually separated connected sets. $\dagger$ If one considers a continuum consisting entirely of cut points and does not impose the restriction that the sets in to which $P$ separates $M$ are connected, then $\mathrm{I}$ have shown that $M$ must be (1) a continuous curve, (2) every point $P$ of $M$ is on an open curve of $M$, and (3) within any circle there are at most a finite number of points from which more than two rays of an open curve depart in $M . \ddagger$

In order that a bounded continuum be an acyclic continuous curve it is necessary and sufficient that any one of the following conditions be satisfied: (a) Every subcontinuum should contain a non-denumerable set of points each of which disconnects $M$ in the strong sense; $\S$ (b) If $K$ denotes the set of all points of $M$ which do not cut $M$, then no subset of $K$ disconnects $M$ in the weak sense; $\|$ (c) The set of all non-cut points shall be totally disconnected in the strong sense. $\llbracket$

* See R. L. Moore, Concerning simple continuous curves, Transactions of this Society, vol. 21 (1920), p. 335.

$\dagger$ See R. L. Moore, On the foundations of plane analysis situs, Transactions of this Society, vol. 17 (1916), p. 159.

¥ See my paper, Closed connected sets that are disconnected by the removal of a finite number of points, Proceedings of the National Academy, vol. 9 (1923), pp. 7-12.

$\S$ See M.C.P.

$\|$ See W. T., loc. cit., Theorem 32, p. 400. This is an extension of a theorem of R. L. Moore, who proved it under the assumption that $M$ is a continuous curve.

I A set of points $K$ is said to be totally disconnected in the strong sense if it does not cease to be totally disconnected upon the addition of a countable set of points. Thus the set of irrational points on a straight line is totally disconnected but fails to be totally disconnected in the strong sense. See C. Zarankiewicz, Z.F., loc. cit., p. 18. 
A continuous curve is said to be two-way continuous if for every pair of points in the set there exist at least two arcs joining the two points, neither of which is a subset of the other; it is cyclically connected if every two points of the set lie together on some simple closed curve of the set. Every cyclically connected continuous curve is two-way continuous but not conversely as may be seen if we consider the set composed of two circles which are tangent externally. In order that a continuous curve may be two-way continuous, it is necessary and sufficient that any one of the following be satisfied: (a) every arc contains a non-cut point or (b) the set of all cut points contains no continuum. It is true that the boundary of every complementary domain of a two-way continuous curve is itself two-way continuous.* The fact that a continuous curve has absolutely no cut points is a necessary and sufficient condition for the curve to be cyclically connected. $\dagger$

5b. Disconnection by Pairs of Points. The best example of a continuous curve that is separated by every pair of points is the simple closed curve. A bounded continuum $M$ will be disconnected by the omission of two of its non-cut points, $A$ and $B$, if and only if there exist two complementary domains such that $A$ and $B$ are both accessible from each of these domains. $\ddagger$ It is of interest to note that for points which go to make up disconnecting pairs, the following theorem similar in most respects to the corresponding theorem for cut points holds: no continuum $M$ has a subcontinuum $K$ which contains an uncountable infinity of points such that if $x$ and $y$ are any two points of $K$, then $M$ but not $K$ is disconnected by the omission of $x$ and $y$. The fact that the boundary of a connected domain contains a

* See G. T. Whyburn, Two-way continuous curves, this Bulletin, vol. 32 (1926), pp. 659-663.

$\dagger$ See G. T. Whyburn, Cyclically connected continuous curves, Proceedings of the National Academy, vol. 13 (1927), Theorem 1, p. 31.

$\ddagger$ See G. T. Whyburn, this Bulletin, vol. 33 (1927), p. 388 . 
noncountable infinity of points such that the omission of any pair of them disconnects the boundary is a necessary and sufficient condition that the boundary be a continuous curve.*

Let us consider a continuum $M$, such that for $M$ there exists a positive integer $k$ such that (1) $M$ is disconnected by the omission of any $k$ of its points but (2) there exists no set of $j[j<k]$ points which disconnects $M$. Every such continuum is a continuous curve. We have previously discussed the case $k=1$. If $k=2$, then $M$ is a simple closed curve. There are no sets that satisfy these conditions for $k>2 . \dagger$ In my result the boundedness is not assumed. If one assumes boundedness then in the case where $k=2$, my condition (1) may be omitted; $\ddagger$ while if we assume that $M$ is connected im kleinen, then in the presence of my conditions (1) and (2) we may dispense with the condition that $M$ is closed and still have the set necessarily a simple closed curve. $\$$

5c. The Menger Curve Theory. Menger reserves the name "curve" for a bounded closed connected set of points $K$ which has the property that for every point $P$ of $K$ and every $e>0$, there exists a neighborhood of diameter less than $e$ about point $P$, whose boundary contains no continuum. As the boundary is closed and contains no continua (other than single points) it follows that the boundary must be zero-dimensional\| and the curve a one-dimensional set if we are dealing with compact metric spaces. In the euclidean plane Menger's curves would consist of those continua

* See G. T. Whyburn, Concerning the disconnection of continua, Fundamenta Mathematicae, vol. 10 (1927).

$\dagger$ See my paper Closed connected sets that are disconnected by the removal of a finite number of points, Proceedings of the National Academy, vol. 9 (1923), pp. 7-12.

‡ See R. L. Moore, Concerning simple continuous curves, loc. cit., p. 342.

§ See R. L. Wilder, this Bulletin, vol. 33 (1927), p. 388.

\| See P. Urysohn, loc. cit., p. 75. 
which contain the interior of no domain.* Thus Menger's "curves" do not include all the sets which are included under the term "continuous curve," where, as is well known, a square and its interior may be included.

If for a point $P$ of the curve $K$, there exists for every $e$ $[e>0]$ a neighborhood about $P$ of diameter less than $e$, whose boundary consists of a finite number of points, then $P$ is called a regular point of the curve. If to a point $P$ of the curve $K$ a number $n$ can be attached so that for every $e$ $[e>0]$, there exists a neighborhood about $P$ of diameter less than $e$ whose boundary consists of not more than $n$ points, then $P$ is said to be of order not greater than $n$. A point is of order exactly $n$, if $n$ is the smallest positive integer with the property that for every preassigned $e$ we may find a neighborhood of diameter less than $e$ whose boundary consists of exactly $n$ points. The regular points to each of which we can assign no positive integer as its order (i.e. the points for which it is possible to find a neighborhood consisting of a finite number of points for every preassigned $e$ but where the number $n$ of boundary points becomes infinite as $e$ approaches zero) will be called points of continually increasing order. In like manner we can define the points of countable order and the points of the order $c$ of the continuum.

Menger proves that (1) the points of order higher than the first are everywhere dense on $K$ and form a set $F_{\sigma}$, which contains continua in every non-vacuous open subset of the curve, (2) the set of end points of $K$ is either vacuous or zerodimensional, (3) in order that a continuum should be a connected im kleinen curve, it is necessary and sufficient that for every $e[e>0], K$ be the sum of a finite number of continua each of diameter less than $e$ and such that no two have a continuum in common; if any two have at most a count-

* In this connection see the abstract of a paper by R. L. Moore in which he shows that a necessary and sufficient condition that a point set shall contain no domain is that for every two points of the set $M$ there exist a totally disconnected subset of $M$ which separates these two points in $M$. See this Bulletin, vol. 32 (1926), p. 218. 
able set in common, then the curve is a continuous curve without any points of order $c .^{*}$

In a later article Menger shows that every regular curve contains for every point $P$ of order $n, n$ arcs which have $P$ as one end point and are otherwise mutually exclusive; for each point of order continually increasing, there are a countable infinity of arcs ending in $P$, otherwise mutually exclusive, and such that the diameters of these arcs can be arranged in a sequence converging to zero. The order of a point of a regular curve is thus the greatest integer $n$ such that there can be found in the curve an acyclic continuous curve consisting of $n$ arcs having $P$ and only $P$ in common. $\dagger$

An interesting complement to the result of Menger just mentioned is a theorem by N. E. Rutt to the effect that if $A$ and $B$ are points of the continuous curve $M$ having the property that it is possible to find $n$ arcs $A X_{i} B(i=1,2$, $3, \cdots, n$ ) having no points in common except $A$ and $B$, then there exist in $M$ a set of points $P_{1}, P_{2}, \cdots, P_{n}$, such that $A$ and $B$ are separated in $M$ by the omission of these points. $\ddagger$

If $K$ is any curve which lies in a compact metric space, then according to Menger there is in euclidean space of three dimensions a continuous curve $M$ which contains a subset which is in (1-1) continuous correspondence with $K . \S$ If the curve $K$ is acyclic, then the set can be found in euclidean space of two dimensions. $\|$

* The above mentioned results are contained in Menger's paper, Grundz ïge einer Theorie der Kurven, Mathematische Annalen, vol. 95, pp. 277-306.

$\dagger$ See Zur allgemeine Kurven Theorie, Fundamenta Mathematicae, vol. 10 (1927), pp. 96-115.

‡ See N. E. Rutt, this Bulletin (abstract), vol. 33 (1927), p. 415.

$\S$ See K. Menger, Allgemeine Räume und Cartesische Räume, Königliche Akademij, Amsterdam, vol. 29, pp. 476-482.

II See M. Wazewski, Sur les courbes de Jordan ne renfermant aucune courbe simple fermée, Annales de la Société Polonaise de Mathématiques, 1924, p. 49. See also H. M. Gehman, Concerning acyclic continuous curves, Transactions of this Society, vol. 29 (1927), Theorem 1. 
5d. The Set $T$ is a Finite (or Countably Infinite) Set of Continua. Most of the characterizations of continuous curves that have been mentioned up to this time, have been based upon the property that certain points or sets of points can be connected in a manner which has been prescribed. However, in 1925, Moore published a characterization, the basic idea of which rests on the ability to separate pairs of points in a certain manner; the result is that a necessary and sufficient condition that a plane continuum be a continuous curve is that, for every two points $A$ and $B$ of the set, there exist a finite number of continua which separate $A$ from $B$ in the set.* According to a theorem due to W. L. Ayres, this disconnection can be made by a set of $n$ arcs, whenever $n$ is the number of maximal connected subsets of $M-(A+B)$ which have $A$ and $B$ both as limit points. $\dagger$

We will recall Miss Mullikin's result to the effect that if $A_{1}, A_{2}, A_{3}, \cdots$ is a countable infinity of closed sets no one of which disconnects its plane $S$, then the sum cannot disconnect $S$. Now, if instead of $S$, we substitute a proper subcontinuum of the plane and let the $A$ 's be subsets of this continuum, then our theorem is no longer true. For example, a circle is not disconnected by the omission of any one of its points but is disconnected by the omission of a countable infinity of them; indeed two suffice to make the separation. Moore, in considering an extension of Miss Mullikin's result, introduced the concept of semi-continuous collections of continua. $\ddagger$

* See R. L. Moore, A characterization of a continuous curve, Fundamenta Mathematicae, vol. 7 (1925), p. 303.

$\dagger$ That the number of such maximal connected subsets is finite follows, with suitable modification, from the previously quoted theorem of Ayres to the effect that if a subcontinuous curve $N$ is subtracted from the continuous curve $M$, then $M-N$ contains at most a finite number of maximal connected subsets of diameter greater than a preassigned $e>0$.

$\ddagger$ If $A$ and $B$ are distinct points, then let $r(A, B)$ denote the distance from $A$ to $B$. If $A$ is a point not in the continuum $q$, then the distance from $A$ to $q$, which we shall denote as $r(A, q)$, shall be the lower bound of all numbers $r(A, Y)$ for all points $Y$ of $q$. If $p$ and $q$ are continua, then the 
Moore proves that, if in a plane $S, M$ is a bounded continuum which does not separate $S$ and $G$ is a countable collection of subcontinua of $M$, no one of which separates $M$, then if the collection is upper semi-continuous, the sum cannot disconnect $M$. He further shows that if $M$ is a continuous curve and two particular points $A$ and $B$ are not separated by any one of the upper semi-continuous collection of subcontinua $G$, then the set of all continua $G$ does not separate $A$ from $B$ in $M .^{*}$ He next considers a plane $S$ every point of which belongs to some continuum of the mutually exclusive set of continua $G$; then, if (1) the collection of continua is upper semi-continuous and (2) no one of them separates the plane, we may regard each continuum as a point and, defining region suitably, we will find that every axiom of his set for plane analysis situs is satisfied, if the space $S$ is interpreted to mean the collection of continua $G$. Thus the set of elements $G$ is topologically equivalent to the set of points of a plane $S . \dagger$

Whyburn takes any plane connected set $M$ and considers the case where $M$ is separated into two mutually separated sets $M_{1}$ and $M_{2}$, by the omission of $n$ of its connected subsets $A_{1}, A_{2}, \cdots, A_{n}$. Then he shows that (1) $M_{i}[i=1,2]+\sum A_{i}$ is the sum of at most $n$ mutually separated connected sets and furthermore (2) if $M_{1}+A_{1}+A_{2}+\cdots+A_{n}$ is the sum

lower distance from $p$ to $q, l(p, q)$, shall be the lower bound [and the upper distance is the upper bound] of the distances $r(X, q)$ for all points $X$ of $p$. A collection of continua $G$ is said to be upper semi-continuous if whenever $p$ is a continuum of the collection $G$ and $p_{1}, p_{2}, p_{3}, \cdots$ is a sequence of continua of $G$ such that the lower distance from $p_{n}$ to $p$ approaches zero as $n$ becomes infinite, then the upper distance from $p_{n}$ to $p$ does so also.

* See R. L. Moore, Concerning upper semi-continuous collections of continua which do not separate the plane, Proceedings of the National Academy, vol. 10 (1924), pp. 356-360.

† See R. L. Moore, Concerning upper semi-continuous collections of continua in the plane, Transactions of this Society, vol. 27 (1925). This concept has also been the basis of extremely interesting work by Alexandroff, Victoria and others. 
of $k$ such sets, then $M_{2}+A_{1}+A_{2} \cdots+A_{n}$ is the sum of at most $n-k+1$ mutually separated connected sets.*

There is an interesting class of connected sets, that are not closed, known as biconnex sets. These sets are the connected sets which cannot be expressed as the sum of two proper subsets which are connected, thus furnishing for connected sets that are not closed an analogue to the indecomposable continua in the closed connected sets. Knaster and Kuratowski gave an extremely interesting example of a biconnex set $B$, having the further property $(b)$ that there is in $B$ a point $P$, such that $B-P$ contains no connected subsets other than single points (a connected set which, we might say, is "exploded" upon the removal of a single point). $\dagger$ Whether every biconnex set contains such an "explosion point" is still unknown; $\ddagger$ every connected set having such a point is biconnex and no biconnex set can have more than one such point. $\S$ A study of sets $H$ belonging to a connected set $K$ and having the property that $K-H$ contains no connected sets other than single points has been made by R. L. Wilder,\| who calls them "dispersion sets."

6. Concluding Remarks. As is evident from the matter presented in this paper, our knowledge of separation properties of sets immersed in three or more dimensions is comparatively limited. Here is an extremely interesting and important field that is practically untouched. Among the various questions that might be considered, we will mention the following.

* See G. T. Whyburn, this Bulletin, vol. 33 (1927), p. 388.

$\dagger$ See Knaster and Kuratowski, Sur les ensembles connexes, Fundamenta Mathematicae, vol. 2 (1921), p. 241.

¥ See Fundamenta Mathematicae, vol. 3, p. 321.

$\S$ See my note, $A$ theorem concerning connected sets, Fundamenta Mathematicae, vol. 3, pp. 238-239.

\| See On dispersion sets of connected sets, Fundamenta Mathematicae, vol. 6, pp. 214-228; see also B. Knaster, Sur un problème de M. R. L. Wilder, in the same journal, vol. 7, pp. 191-198. 
(a) The problem of finding approachability conditions which are necessary and sufficient that the separating set be a simple closed surface, i.e., the set of points that can be put into (1-1) continuous correspondence with the surface of a sphere.

(b) The problem of securing necessary and sufficient conditions that the sum of two closed connected sets, neither of which separates three-space, may have a sum that separates three-space (the conditions of Mazurkiewicz and Strasziewicz mentioned above are sufficient but not necessary).

(c) The question of when a continuous (1-1) correspondence $T$ between two simple closed surfaces in three dimensions, may be extended to a (1-1) continuous correspondence $\bar{T}$ of their surrounding three-spaces such that $T$ and $\bar{T}$ are identical for points of the surfaces given.*

(d) The problem of finding conditions analogous to those of Schoenflies that are necessary and sufficient that a continuum in three dimensions be a continuous curve. The conditions of Schoenflies might be characterized as external conditions in that they give the relation of the continuum to the surrounding space. The connectivity im kleinen condition of Hahn, the $S$ property of Sierpinski, the requirement of Wilder that every connected subset of an open subset be arcwise connected, and Moore's separation condition are all of them internal conditions and hold in any number of dimensions. The problem of finding external conditions is an open one. $\dagger$

We do not wish, however, to create the impression that the problems of two dimensions are by any means completely solved. An interesting set of problems among those still

* That this extension is not always possible has been shown by $\mathrm{J} . \mathrm{W}$. Alexander by means of an interesting example in the Proceedings of the National Academy, vol. 10 (1924).

$\dagger$ For a discussion of the difficulties encountered see the article of R. L. Moore, Concerning the relation of a continuous curve to its complement in three dimensions, loc. cit. 
unsolved is concerned with the question as to whether every continuum such that every subcontinuum, containing more than one point, is homeomorphic with the whole set must be a simple continuous arc and whether there are bounded homogeneous plane continua which are not simple closed curves.* A solution of the various problems still unsolved in two and three dimensions will open up tremendous possibilities for future work in this important and fascinating branch of mathematics.

The University of Pennsylvania

* That such continua, if they exist, cannot be continuous curves follows from the results of Mazurkiewicz, Sur les continus homogènes, Fundamenta Mathematica, vol. 5 (1924), pp. 137-146. A set $M$ is said to be homogeneous if for every two points $x$ and $y$ of $M$ there exists a (1-1) continuous transformation of $M$ into itself which turns $x$ into $y$. 